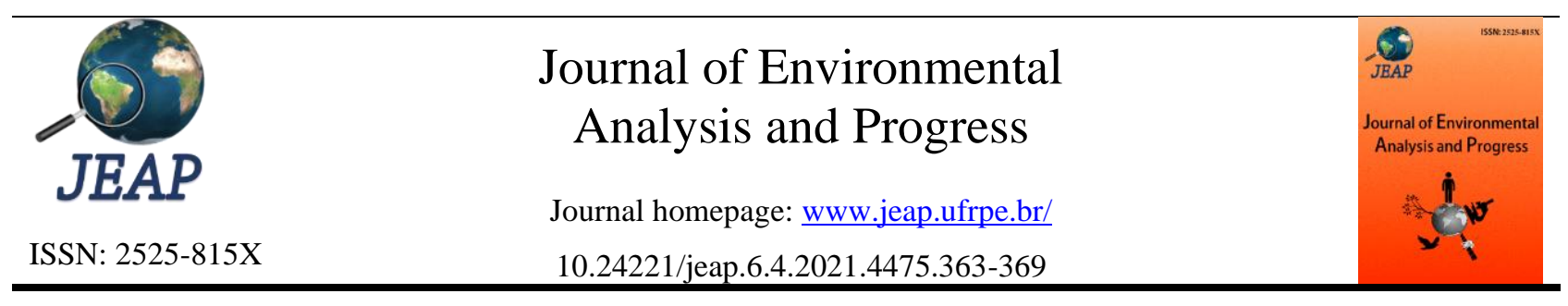

\title{
Construção e validação de uma mesa de tensão de baixo custo
}

\section{Construction and validation of a low-cost tension table}

Samuel Dias Moreira ${ }^{\mathrm{a}}$, André Cabral França ${ }^{\mathrm{b}}$, Wellington Willian Rocha ${ }^{\mathrm{c}}$, Mucio Magno de Melo Farnezi $^{\mathrm{d}}$, Fabrício Resende de Aguiar ${ }^{\mathrm{e}}$

${ }^{a}$ Universidade Federal dos Vales do Jequitinhonha e Mucuri-UFVJM, Departamento de Ciências Agrárias, Campus JK. Rodovia MGT 367, Km 683, $\mathrm{n}^{\circ}$ 5.000, Alto da Jacuba, Diamantina-MG, Brasil. CEP: 39100-000. E-mail: agronomia_samuel@yahoo.com.br, cabralfranca@gmail.com, wwillian@ufvjm.edu.br, muciomagno@yahoo.com.br, fabricio.r.a1996@gmail.com.

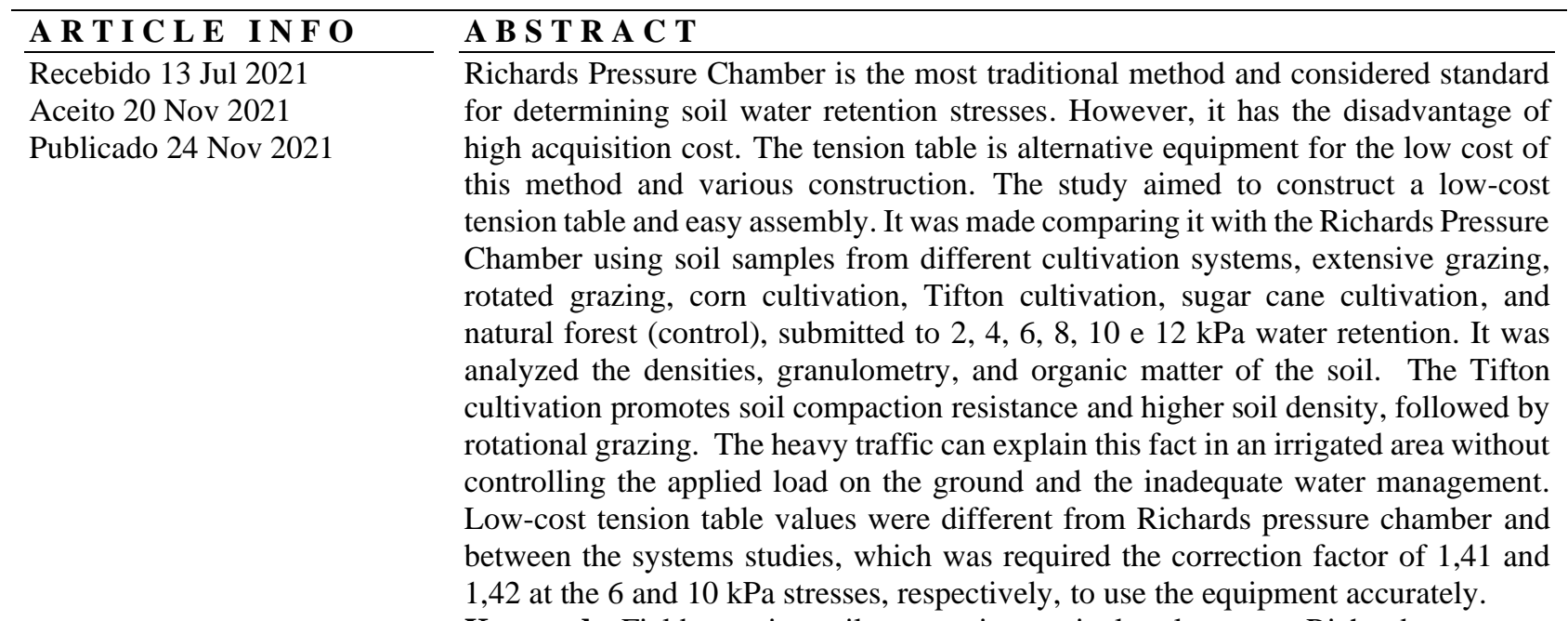

Keywords: Field capacity, soil compaction, agricultural systems, Richards extractor.

\section{R E S U M O}

A câmara de pressão de Richards é o método mais tradicional e considerado padrão para determinar as tensões de retenção de água no solo, porém detém da desvantagem do elevado custo de aquisição. A mesa de tensão é um equipamento alternativo para este método, sendo de baixo custo e construção variada. Objetivou-se neste estudo construir uma mesa de tensão de baixo custo e fácil montagem para determinação da tensão de retenção de água no solo, comparando-a posteriormente à câmara de pressão de Richards utilizando amostras de solo de diferentes sistemas de cultivo, pastejo extensivo, pastejo rotacionado, cultivo de milho, cultivo de tifton, cultivo de cana-de-açúcar e mata natural (controle), submetidas às tensões de água de 2, 4, 6, 8, 10 e $12 \mathrm{kPa}$. Foram analisadas densidade, granulometria e teor de matéria orgânica do solo. A área sob cultivo de tifton, mesmo com o maior teor de matéria orgânica, promotor da resistência do solo à compactação, apresentou maior densidade do solo, seguida pelo pastejo rotacionado. Este fato pode ser explicado pelo intenso tráfego de máquinas em área irrigada sem o controle da carga aplicada no solo e o manejo inadequado da água. Os valores obtidos da mesa de tensão de baixo custo foram diferentes daqueles da câmara de pressão de Richards e entre os sistemas estudados, sendo necessário a aplicação do fator de correção de 1,41 e 1,42 nas tensões de 6 e $10 \mathrm{kPa}$, respectivamente, para utilizar o equipamento com precisão.

Palavras-Chave: Capacidade de campo, compactação, sistemas agrícolas, extrator de Richards. 


\section{Introdução}

A curva de retenção de água é uma das variáveis relacionadas à propriedade hidráulica do solo, desempenhando papel de destaque no transporte de solutos (Shwetha \& Varija, 2015), na movimentação de água, no desenvolvimento de plantas e na regulação da dinâmica da umidade do solo (Gao et al., 2018).

A curva de retenção de água relaciona a quantidade de água com a energia proveniente de sua retenção no solo (Filgueiras et al., 2016). Em solos não saturados, essa curva é uma ferramenta crucial na descrição do comportamento físicoquímico e na mecânica desses solos (Lucas et al., 2011). Na irrigação é possível, por meio desse comportamento, estimar a disponibilidade de água no solo para as plantas e definir o ponto de murcha permanente, bem como a capacidade de campo do solo (Filgueira et al., 2016).

A capacidade de campo destaca-se no manejo de irrigação, pois permite aplicar uma lâmina de água sem excesso de lixiviação e definir o estado hídrico do solo (Lier, 2017). De acordo com sua definição clássica, "é a quantidade de água retida pelo solo depois que o excesso de água foi drenado e a taxa de movimento descendente da água foi decrescido acentuadamente, o que, geralmente, ocorre dentro de 2 ou 3 dias após uma chuva ou irrigação no solo permeável de estrutura e textura uniforme" (Veihmeyer \& Hendrickson, 1949).

Esse conceito clássico da capacidade de campo refere-se à máxima quantidade de água que um solo consegue reter após a saturação e a drenagem do excesso de água, atingindo um equilíbrio com o mínimo de lixiviação. Mas, colocar isso em prática é muito complexo, trabalhoso e demorado, além de ser um conceito arbitrário. No entanto, tensões próximas de $6 \mathrm{kPa}$ foram encontradas utilizando-se o ponto de inflexão para calcular a capacidade de campo para Latossolos (Mello et al., 2002).

As câmaras de pressão com placa porosa (Richards, 1949) e os funis de placa porosa (Haines, 1930) são equipamentos utilizados na determinação da curva de retenção de água no solo. A câmara de pressão de Richards é o método mais tradicional e considerado padrão, porém, tem a desvantagem do elevado custo de aquisição (Lucas et al., 2011; Melo Filho, Sacramento \& Conceição, 2015). A mesa de tensão também é empregada para a determinação da capacidade de campo, exclusivamente para baixas tensões. Esse equipamento tem como vantagens a facilidade na montagem, a flexibilidade na construção, a fácil utilização e o baixo custo, como, por exemplo, as colunas de areia (Reinert \& Reichert, 2006).
Este estudo foi realizado com o objetivo de construir uma mesa de tensão de baixo custo e fácil montagem para determinar a tensão de retenção de água no solo. Posteriormente, buscou-se validar a mesa de tensão construída, comparando-a com a câmara de pressão de Richards, por meio de modelos matemáticos lineares utilizando-se amostras de solo de diferentes sistemas de cultivo.

\section{Material e Métodos}

Construção da mesa de tensão

Uma estrutura de madeira com altura de $150 \mathrm{~cm}$ foi construída para apoiar a mesa de tensão, sendo o comprimento e a largura baseados nas dimensões da bandeja utilizada como recipiente das amostras de solo (Figura 1A). Utilizou-se uma bandeja de plástico com $48 \mathrm{~cm}$ de comprimento, 28 $\mathrm{cm}$ de largura e $8,5 \mathrm{~cm}$ de altura, entretanto, outras bandejas e dimensões podem ser utilizadas, desde que o recipiente seja rígido e impermeável (Figura 1B). A bandeja foi encaixada na parte superior dessa estrutura e conectada a tubulações de policloreto de vinila (PVC). No meio da bandeja foi feito um furo para inserir um "adaptador para caixa d'água de $50 \mathrm{~mm}$ " e, por meio de conexões, uma "redução excêntrica 100 x $50 \mathrm{~mm}$ " foi encaixada e conectada a um tubo de $100 \mathrm{~mm}$ de diâmetro (Figura 1C).

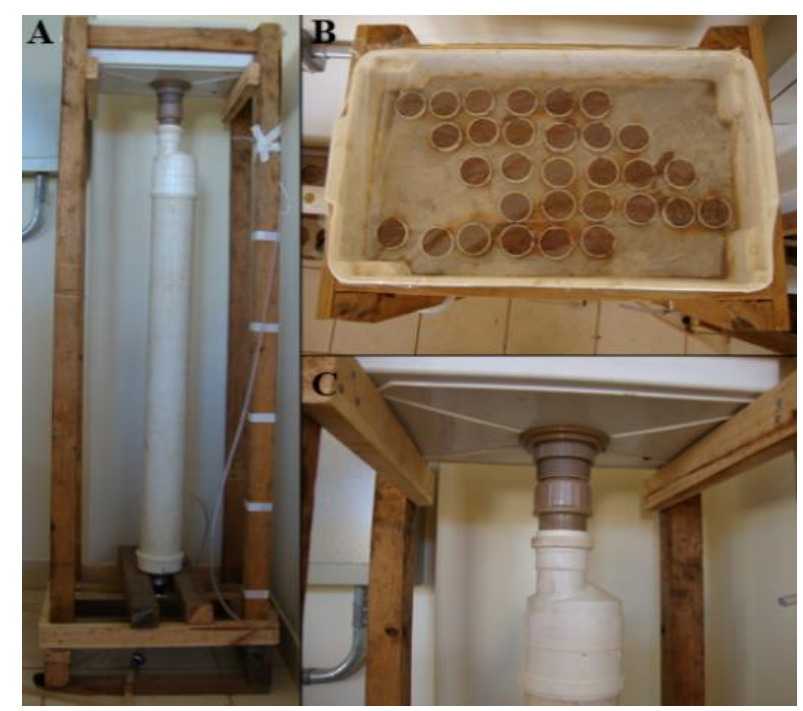

Figura 1. Mesa de tensão construída em colunas de policloreto de vinila (PVC) com areia. A. Vista geral do equipamento; B. bandeja com amostras de solo; C. detalhes das conexões de PVC. Fonte: Moreira et al. (2021).

Na parte inferior da coluna de PVC foi acoplado um tampão e, no meio dele, um "adaptador para caixa d'água de $20 \mathrm{~mm}$ "; posteriormente, um "adaptador de mangueira 1/2" foi colocado através de conexões para o abastecimento de água. Na parte inferior também 
foi acoplado um microtubo flexível para saída de água com comprimento que atingisse a altura da bandeja. A coluna de PVC deve ter um comprimento total de $120 \mathrm{~cm}$ e seu interior preenchido com brita no terço inferior, areia grossa no terço médio e areia média no terço superior. $\mathrm{O}$ tamanho das partículas no interior da coluna aumenta de baixo para cima. Na bandeja foi colocada uma camada de $2 \mathrm{~cm}$ de areia fina e, no topo, um papel filtro.

\section{Funcionamento}

Uma mangueira foi conectada no abastecimento de água, na parte inferior da coluna, com o objetivo de encher a mesa de tensão de baixo para cima e de forma lenta, para evitar bolhas de ar no interior da coluna. $\mathrm{O}$ abastecimento de água foi desligado quando a água encobriu a areia da bandeja e drenada completamente para assentamento das partículas dentro da coluna.

A partir da altura média da amostra de solo posicionada na bandeja, a estrutura de madeira foi graduada de $20 \mathrm{em} 20 \mathrm{~cm}$, até totalizar $120 \mathrm{~cm}$; cada $20 \mathrm{~cm}$ de comprimento equivale a $2 \mathrm{kPa}$ de tensão. $\mathrm{O}$ funcionamento da mesa de tensão consiste na diferença de nível da amostra e o posicionamento do tubo flexível de saída de água, proporcionando uma sucção da água. Esse funcionamento obedeceu ao mesmo princípio proposto por Lima \& Silva (2008), com coluna de água realizando a sucção até atingir o equilíbrio hidrostático.

As amostras de solo saturadas foram colocadas na mesa de tensão, garantindo o contato da amostra com a superfície do papel filtro. A bandeja foi coberta com um filme plástico para evitar a perda de água por evaporação. As tensões aplicadas variam de 0 a $12 \mathrm{kPa}$ e consistem em determinar as umidades gravimétricas do solo em suas respectivas tensões de retenção de água. $\mathrm{O}$ equilíbrio hidrostático para cada tensão demonstrou-se alcançado ao notar-se a suspensão do gotejo da água no tubo flexível de saída.

\section{Descrições dos locais de estudo}

O estudo foi realizado em diferentes locais na região de Lagoa da Prata, Estado de Minas Gerais, Brasil $\left(20^{\circ} 01^{\prime} 22^{\prime \prime} \mathrm{S}, 45^{\circ} 32^{\prime} 38^{\prime \prime} \mathrm{O}\right.$, altitude de $650 \mathrm{~m}$ ).

Os solos e seus respectivos sistemas de cultivo na área de estudo foram Latossolo Vermelho-Amarelo distrófico (EMBRAPA, 2013). sob pastejo extensivo, cuja área era cultivada com pastagem há cerca de 30 anos, sem realizar preparo do solo, e o manejo feito de forma contínua, sem o controle da carga que o animal suporta, utilizada por animais leves (recria). Nessa área não foi realizada adubação desde sua implantação e, apesar do manejo inadequado, apresentou-se com poucos sinais de degradação. Os demais solos foram Latosssolos Vermelhos distroférricos (EMBRAPA, 2013) nos seguintes sistemas de cultivo: cultivo de milho, cultivo de tifton (Cynodon spp.), cultivo de cana-de-açúcar, pastejo rotacionado com Urochloa brizantha (Syn. Brachiaria brizantha) e mata natural.

A área do cultivo de milho apresentava-se infestada por Urochloa spp. (Syn. Brachiaria spp.) e, no momento da colheita mecanizada, formou-se uma cobertura morta espessa. Historicamente, nessa área cultivaram-se cana-de-açúcar e arroz, durante 10 anos, e, no último ano, cultivou-se o milho em sistema convencional. O cultivo de tifton foi realizado sob pivô central e utilizado maquinário pesado para a produção de feno. Esse manejo foi realizado durante 14 anos e nunca passou por avaliações físicas do solo e práticas para descompactação do solo. A área de pastagem foi formada há 19 anos e, nos últimos três anos, adotou-se o sistema de pastejo rotacionado. O pasto foi formado com Urochloa brizantha cv. MG 5 e adubada, anualmente, para a manutenção da fertilidade.

$\mathrm{Na}$ área do cultivo da cana-de-açúcar foram feitas três colheitas de forma manual sem realizar a queima da palhada. O material colhido foi retirado com trator de pneus de pequeno porte e carreta de duas rodas. Este tipo de manejo ajuda a aumentar a matéria orgânica do solo e o protege contra a erosão hídrica. Nessa área, o milho cultivado por três anos foi sucedido pela cana-deaçúcar. A mata foi o tratamento controle, sistema natural sem intervenção antrópica, caracterizada como mata de sucupira típica de transição dos biomas de Cerrado para floresta semidecidual da Mata Atlântica.

Em relação às coletas e análises dos solos estudados, as amostras com estruturas deformadas e indeformadas foram coletadas em cada sistema de cultivo na camada de 0 a $20 \mathrm{~cm}$. As amostras indeformadas foram coletadas utilizando-se $\mathrm{o}$ amostrador de Uhland. Para cada sistema de cultivo foram coletadas 10 amostras de solos identificadas e levadas para o laboratório. As análises granulométricas pelo método da pipeta (Day, 1965; EMBRAPA, 1997), as densidades do solo (DS) pelo método dos anéis volumétricos (Blake \& Hartge, 1986) e os teores de matéria orgânica (MO) (Raij \& Quaggio, 1983) foram determinadas no laboratório. As médias da DS e da MO foram comparadas entre os sistemas de cultivo pelo teste $\mathrm{T}$ de student, a $5 \%$.

As amostras deformadas foram peneiradas em malha de $2 \mathrm{~mm}$ e acondicionadas dentro de 
anéis identificados. Os anéis foram feitos de tubos de PVC com diâmetro de $32 \mathrm{~mm}$ e $20 \mathrm{~mm}$ de altura. Os solos foram saturados em água destilada por 72 horas e, posteriormente, colocados na mesa de tensão (Figura 1B).

A mesa de tensão foi comparada com a câmara de pressão de Richards (Richards, 1949) para sua validação. As amostras de solo foram submetidas às tensões de $2,4,6,8,10$ e $12 \mathrm{kPa}$, com dez repetições para cada sistema de cultivo, totalizando 60 parcelas para cada equipamento. As amostras na mesa de tensão foram conduzidas ao mesmo tempo. Para tal, fez-se necessária a construção de duas mesas, dado o grande número de parcelas experimentais. A divisão das amostras nas duas mesas foi equivalente, contendo todos os sistemas em estudo, cada qual com cinco repetições.

Os resultados dos equipamentos foram comparados seguindo o procedimento descrito por Snedecor \& Cochran (1989), plotando-se as curvas de retenção de água. $\mathrm{O}$ modelo $\mathrm{y}=\mathrm{a} \mathrm{xb}$ foi ajustado às curvas de retenção de cada tratamento. Para compararem-se as curvas, procedeu-se à linearização $[\ln (\mathrm{y})=\ln (\mathrm{a})+\mathrm{b} \ln (\mathrm{x})]$, comparando-se os coeficientes linear e angular entre os tratamentos. Primeiramente, as mesas de tensão foram comparadas e sua concordância resultou na união dos resultados. Posteriormente, a mesa de tensão e a câmara de pressão de Richards foram comparadas estatisticamente.

Determinou-se, também, a razão entre os equipamentos nas tensões de 6 e $10 \mathrm{kPa}$, definindose um fator de correção para cada sistema de cultivo. Os resultados obtidos para as amostras dos diferentes sistemas de cultivo sob análise na câmara de pressão de Richards foram comparados pelo teste Tukey (5\%).

\section{Resultados e Discussão}

Os sistemas de cultivo apresentaram diferentes texturas (Tabela 1), teor de matéria orgânica e densidade do solo (Tabela 2). A área sob cultivo de tifton, mesmo com o maior teor de matéria orgânica, promotor da resistência do solo à compactação, apresentou maior densidade do solo, seguida pelo pastejo rotacionado. Este fato pode ser explicado pelo intenso tráfego de máquinas em área irrigada sem o controle da carga aplicada no solo e manejo inadequado da água.

O tráfego das máquinas agrícolas proporciona aumento na densidade do solo (Roque et al., 2010) e os solos úmidos e de alto teor de argila são mais vulneráveis ao impacto dessa compactação (Stoessel et al., 2018).
Tabela 1. Textura do solo dos diferentes sistemas de cultivo. Fonte: Moreira et al. (2021).

\begin{tabular}{lccc}
\hline \multirow{2}{*}{ Sistemas } & Areia & Silte & Argila \\
\hline Milho & $------\mathbf{g ~ k g}^{-1}$ & $--\cdot----$ \\
Tifton & 13 & 29 & 59 \\
Pastejo rotacionado & 4 & 37 & 59 \\
Pastejo extensivo & 11 & 42 & 54 \\
Cana-de-açúcar & 13 & 25 & 64 \\
Mata (controle) & 10 & 28 & 60 \\
\hline
\end{tabular}

Tabela 2. Teor de matéria orgânica (MO) e densidade (DS) do solo dos diferentes sistemas de cultivo. Fonte: Moreira et al. (2021).

\begin{tabular}{lcc}
\hline Sistemas & $\mathbf{M O}$ & $\mathbf{D S}$ \\
\hline Milho & $\mathbf{d a g} \mathbf{~ k g}^{-1}$ & $\mathbf{g ~ c m}^{-3}$ \\
Tifton & $19 \mathrm{~b}$ & $1,16 \mathrm{c}$ \\
Pastejo rotacionado & $32 \mathrm{a}$ & $1,43 \mathrm{a}$ \\
Pastejo extensivo & $24 \mathrm{~b}$ & $1,32 \mathrm{~b}$ \\
Cana-de-açúcar & $16 \mathrm{~b}$ & $1,11 \mathrm{c}$ \\
Mata (controle) & $17 \mathrm{~b}$ & $1,05 \mathrm{~d}$ \\
\hline
\end{tabular}

Médias seguidas de mesma letra na coluna, não são diferentes entre si, pelo teste $\mathrm{T}$ de student, a $5 \%$.

O excesso de carga animal para o pastejo rotacionado, quando ainda associada a uma pressão inapropriada aplicada, pode ocasionar maior densidade do solo. O pisoteio animal e o tráfego de máquinas agrícolas em faixa inadequada de umidade do solo são as principais causas da compactação do solo (Pires et al., 2012; Neiva Júnior et al., 2015). Alterações na densidade do solo afetam o movimento da água, dos nutrientes e o crescimento de raízes e, consequentemente, influenciam o desenvolvimento das pastagens (Souza et al., 2013).

Fez-se a comparação entre as duas mesas de tensão utilizando-se de modelos matemáticos lineares (Snedecor \& Cochran, 1989) ajustados para as umidades do solo e as tensões de retenção de água (Tabela 3). Não houve diferença estatística significativa entre os modelos gerados para as duas mesas de tensão independente do sistema de cultivo. Assim, as duas mesas de tensão foram utilizadas na validação do equipamento, pois mostram similaridade dos resultados.

Tabela 3. Modelos lineares ajustados para os diferentes sistemas de cultivo nas diferentes tensões de retenção de água no solo, comparandose duas mesas de tensão igualmente construídas. Fonte: Moreira et al. (2021).

\begin{tabular}{lccc}
\hline $\begin{array}{l}\text { Sistemas } \\
\text { de cultivo }\end{array}$ & \multicolumn{2}{c}{ Equação ajustada } \\
\hline Mesa 1 & Mesa 2 & \\
\hline & $y=-0,458 x+$ & $y=-0,488 x$ & NS \\
& 29,571 & $+31,992$ &
\end{tabular}




\begin{tabular}{lccc}
\hline & $\mathrm{R}^{2}=0,80$ & $\mathrm{R}^{2}=0,78$ & \\
Tifton & $\mathrm{y}=-0,501 \mathrm{x}+$ & $\mathrm{y}=-0,516 \mathrm{x}$ & $\mathrm{NS}$ \\
& 30,156 & $+32,129$ & \\
& $\mathrm{R}^{2}=0,61$ & $\mathrm{R}^{2}=0,71$ & \\
P. & $\mathrm{y}=-0,624 \mathrm{x}+$ & $\mathrm{y}=-0,651 \mathrm{x}$ & $\mathrm{NS}$ \\
rotacionado & 28,238 & $+27,047$ & \\
& $\mathrm{R}^{2}=0,72$ & $\mathrm{R}^{2}=0,77$ & \\
P. & $\mathrm{y}=-0,405 \mathrm{x}+$ & $\mathrm{y}=-0,433 \mathrm{x}$ & $\mathrm{NS}$ \\
extensivo & 22,985 & $+21,956$ & \\
& $\mathrm{R}^{2}=0,80$ & $\mathrm{R}^{2}=0,90$ & \\
Cana-de- & $\mathrm{y}=-0,405 \mathrm{x}+$ & $\mathrm{y}=-0,444 \mathrm{x}$ & $\mathrm{NS}$ \\
açúcar & 22,985 & $+25,501$ & \\
& $\mathrm{R}^{2}=0,80$ & $\mathrm{R}^{2}=0,76$ & \\
Mata & $\mathrm{y}=-0,371 \mathrm{x}+$ & $\mathrm{y}=-0,352 \mathrm{x}$ & $\mathrm{NS}$ \\
(controle) & 22,513 & $+22,724$ & \\
& $\mathrm{R}^{2}=0,78$ & $\mathrm{R}^{2}=0,79$ & \\
\hline
\end{tabular}

NS Não significativo, pelo método de Snedecor \& Cochran (1989) e (P) pastejo.

Os resultados das duas mesas de tensão foram combinados e novas equações foram ajustadas para cada sistema de cultivo. A partir dessas novas equações, a mesa de tensão foi comparada à câmara de pressão de Richards por meio de modelos lineares (Snedecor \& Cochran, 1989) (Tabela 4). Os modelos lineares gerados da mesa de tensão apresentaram diferença estatística dos modelos gerados pela câmara de pressão de Richards. A câmara de pressão de Richards é considerada precisa e comercialmente utilizada, dada a sua constituição por placas porosas com granulometria uniforme e com mínima deformabilidade, garantindo uma aplicação de pressão constante.

Tabela 4. Modelos lineares ajustados para os diferentes sistemas de cultivo nas diferentes tensões de retenção de água no solo, comparandose a mesa de tensão construída com a câmara de pressão de Richards. Fonte: Moreira et al. (2021).

\begin{tabular}{|c|c|c|c|}
\hline \multirow[b]{2}{*}{$\begin{array}{l}\text { Sistemas de } \\
\text { cultivo }\end{array}$} & \multicolumn{3}{|c|}{ Equação ajustada } \\
\hline & $\begin{array}{l}\text { Mesa de } \\
\text { tensão }\end{array}$ & $\begin{array}{c}\text { Câmara de } \\
\text { pressão }\end{array}$ & \\
\hline Milho & $\begin{array}{l}y=-0,46 x+ \\
27,82 R^{2}= \\
0,77\end{array}$ & $\begin{array}{c}\mathrm{y}=-0,93 \mathrm{x}+ \\
31,99 \mathrm{R}^{2}= \\
0,77\end{array}$ & \\
\hline Tifton & $\begin{array}{l}y=-0,52 x+ \\
28,61 \quad R^{2}= \\
0,63\end{array}$ & $\begin{array}{c}y=-1,11 x+ \\
38,82 \mathrm{R}^{2}= \\
0,82\end{array}$ & ** \\
\hline $\begin{array}{l}\mathrm{P} . \\
\text { rotacionado }\end{array}$ & $\begin{array}{l}y=-0,54 x+ \\
29,68 \quad R^{2}= \\
0,69\end{array}$ & $\begin{array}{c}y=-0,99 x+ \\
35,04 R^{2}= \\
0,76\end{array}$ & $* *$ \\
\hline P. exte & $\begin{array}{l}y=-0,46 x+ \\
25,61 \quad R^{2}= \\
0,76\end{array}$ & $\begin{array}{c}y=-0,94 x+ \\
33,75 R^{2}= \\
0,85\end{array}$ & $* *$ \\
\hline
\end{tabular}

Cana-de-

açúcar

Mata

(controle)

${ }^{* *}$ Significativo, a 5\%, pelo método de Snedecor \& Cochran (1989) e (P) pastejo.

Os sistemas de cultivos foram comparados dois a dois por meio dos modelos lineares (Snedecor \& Cochran, 1989) para cada equipamento, mesa de tensão e câmara de pressão de Richards (Tabela 5). Observa-se que as mesmas diferenças estatísticas entre os sistemas foram encontradas nos dois equipamentos, sendo elas cultivo de tifton versus pastejo rotacionado e pastejo extensivo versus cultivo de cana-de-açúcar, que não apresentaram diferenças estatísticas entre si, e os demais foram todos estatisticamente diferentes. Isto demonstra que, apesar de os dois equipamentos apresentarem resultados diferentes, eles exibem uma mesma tendência.

Tabela 5. Significância entre os modelos ajustados para os diferentes sistemas de cultivo na mesa de tensão e na câmara de pressão de Richards. $\mathrm{P}=$ pastejo. Fonte: Moreira et al. (2021).

\begin{tabular}{|c|c|c|}
\hline \multirow[b]{2}{*}{ Sistemas de cultivo } & \multicolumn{2}{|c|}{ Significância } \\
\hline & $\begin{array}{c}\text { Mesa } \\
\text { de } \\
\text { tensão }\end{array}$ & $\begin{array}{l}\text { Câmar } \\
\text { a de } \\
\text { pressão }\end{array}$ \\
\hline Milho $\mathrm{x}$ tifton & *** & *** \\
\hline Milho x p. rotacionado & ** & ** \\
\hline Milho x p. extensivo & ** & ** \\
\hline Milho x cana-de-açúcar & *** & *** \\
\hline Milho x mata & *** & ** \\
\hline Tifton $\mathrm{x}$ p. rotacionado & NS & NS \\
\hline Tifton $\mathrm{x}$ p. extensivo & *** & ** \\
\hline Tifton x cana-de-açúcar & *** & *** \\
\hline Tifton $\mathrm{x}$ mata & ** & ** \\
\hline $\begin{array}{l}\text { P. rotacionado x p. } \\
\text { extensivo }\end{array}$ & ** & ** \\
\hline $\begin{array}{l}\text { P. rotacionado x cana-de- } \\
\text { açúcar }\end{array}$ & ** & ** \\
\hline P. rotacionado x mata & ** & ** \\
\hline $\begin{array}{l}\text { P. extensivo x cana-de- } \\
\text { açúcar }\end{array}$ & NS & NS \\
\hline P. extensivo $\mathrm{x}$ mata & ** & ** \\
\hline Cana-de-açúcar x mata & ** & ** \\
\hline
\end{tabular}

NS Não significativo, ${ }^{* *}$ Significativo a $5 \%$, pelo método de Snedecor \& Cochran (1989).

Na câmara de pressão de Richards observase que as umidades foram maiores para os manejos tifton e pastejo rotacionado. Estes sistemas de cultivo também apresentam maiores valores de 
densidade do solo que, por consequência, aproximam as partículas do solo reduzindo a percolação da água, conservando a água mais tempo no sistema solo. Outro fato para a maior retenção de água para estes solos é que eles apresentam maiores conteúdos de matéria orgânica, que têm grande capacidade de reter água (Rocha et. al, 2002).

Tabela 6. Fatores de correção para validação do protótipo nas tensões de retenção de água no solo de 6 e $10 \mathrm{kPa}$ em diferentes sistemas de cultivo. Fonte: Moreira et al. (2021).

\begin{tabular}{lcc}
\hline \multirow{2}{*}{ Sistemas de cultivo } & \multicolumn{2}{c}{ Fator de correção } \\
\cline { 2 - 3 } & $\mathbf{6 ~ k P a}$ & $\mathbf{1 0 ~} \mathbf{~ P a}$ \\
\hline Milho & 1,54 & 1,51 \\
Tifton & 1,33 & 1,45 \\
P. rotacionado & 1,36 & 1,47 \\
P. extensivo & 1,41 & 1,36 \\
Cana-de-açúcar & 1,32 & 1,31 \\
Mata (controle) & 1,43 & 1,44 \\
\hline Média & 1,41 & 1,42 \\
\hline
\end{tabular}

(P) pastejo.

Os fatores de correção se apresentaram de forma variável para cada sistema, pois como não se trata de apenas um solo e um único sistema, a relação não foi constante (Tabela 6). $\mathrm{O}$ fator em questão variou entre 1,32 a 1,54 para validação do equipamento na tensão de $6 \mathrm{kPa}$, e 1,31 a 1,51 sob tensão de $10 \mathrm{kPa}$, valores este correspondentes ao sistema de cultivo com cana-de-açúcar e milho, de modo respectivo.

Para o pastejo rotacionado e extensivo, os coeficiente foram de 1,36 e 1,41 na tensão de $6 \mathrm{kPa}$, e 1,47 e 1,36 na tensão de $10 \mathrm{kPa}$, na devida ordem. O solo da área sob cultivo com Tifton, por sua vez, apresentou constantes de correção de 1,33 e 1,45 à $6 \mathrm{kPa}$ e $10 \mathrm{kPa}$, respectivamente. O solo sob mata natural apresentou os valores de 1,43 e 1,44 sob tensão de 6 e $10 \mathrm{kPa}$, nesta ordem. Por fim, foram verificados valores médio dos fatores de correção de 1,41 para validação do equipamento à tensão de retenção de água no solo de $6 \mathrm{kPa}$, e 1,42 para a tensão de $10 \mathrm{kPa}$, respectivamente.

A variabilidade dos fatores de correção ocorre devido à condutividade hidráulica dentro da mesa de tensão, dada a desuniformidade da areia, ainda que peneirada, quando comparada a placa porosa da câmara de pressão de Richards. Dessa forma, ocorre variação no fluxo de água e, possivelmente, sua retenção em função da pressão aplicada. $\mathrm{O}$ fator de correção foi necessário para utilizar o equipamento com precisão.

\section{Conclusão}

A mesa de tensão construída em colunas de PVC com areia foi de baixo custo, de fácil montagem e manuseio, e viável para determinar a retenção de água no solo em baixas tensões, requerendo apenas a calibração dos valores de umidade obtidos para utilizar o equipamento com precisão.

A média obtida para o fator de correção foi de 1,41 e 1,42 nas tensões de retenção de água no solo de 6 e $10 \mathrm{kPa}$, respectivamente.

\section{Agradecimentos}

Os autores agradecem o apoio da Coordenação de Aperfeiçoamento de Pessoal de Nível Superior - Brasil (CAPES) - Código de Financiamento 001.

\section{Referências}

Blake, G. R.; Hartge, K. H. 1986. Bulk density. In: Klute A, editor. Methods of soil analysis. 2 ed. Madison: American Society of Agronomy. Part 1, pp. 363-375.

Day, P. R. 1965. Particle fractionation and particlesize analysis. In: Black, C. A.; Evans, D. D.; Ensminger, L. E.; White, J. L.; Clark, F. E. (ed.). Methods of soil analysis. Madison: American Society of Agronomy, pp. 545-567.

EMBRAPA. 1997. Manual de métodos de análise de solo. 2 ed. Brasília, DF: Centro Nacional de Pesquisas do Solo. 212p.

EMBRAPA. 2013. Sistema Brasileiro de Classificação de Solos. 3 ed. Brasília. 353p.

Filgueira, R; Oliveira, V. M. R. de; Cunha, F. F. da; Mantovani, E. C; Souza, E. J. de. 2016. Modelos de curva de retenção de água no solo. Irriga, Edição Especial, Irriga \& Inovagri, 1, 1, 115-120.

Gao, Z.; Wang, Y.; Luo, J.; Liu, M. 2018. Rootinduced changes to soil water retention in permafrost regions of the Qinghai-Tibet Plateau, China. Journal of Soils and Sediments, 18, 3, 791-803.

Haines, W. B. Studies in the physical properties of soil. 1930. V. The hysteresis effect in capillary properties, and the modes of moisture distribution associated therewith. The Journal of Agricultural Science, 20, 1, 97-116.

Lier, Q. D. J. V. 2017. Field capacity, a valid upper limit of crop available water? Agricultural Water Management, 193, 214-220.

Lima, H. V.; Silva, A. P. 2008. Mesa de tensão com areia: procedimentos para montagem e validação. Revista Brasileira de Ciência do Solo, 32, 5, 2209-2214. 
Lucas, J. F. R.; Tavares, M. H. F.; Décio, L. C.; Cássaro, F. A. M. 2011. Curva de retenção de água no solo pelo método do papel-filtro. Revista Brasileira de Ciências do Solo, 35, 6, 1957-1973.

Mello, C. R. 2002. Estimativa da capacidade de campo baseada no ponto de inflexão da curva característica. Ciência e Agrotecnologia, 26, 4, 836-841.

Melo Filho, J. F.; Sacramento, J. A. A. S.; Conceição, B. P. S. 2015. Curva de retenção de água elaborada pelo método do psicrômetro para uso na determinação do índice "S" de qualidade física do solo. Engenharia Agrícola, 35, 5, 959-966.

Neiva Júnior, E.; Rocha, W. W.; Pires, B. S.; Farnezi, M. M. de M.; Dias Junior, M. de S.; Freitas, D. F. B. de; Silva, E. B.; Carvalho, G. A. de O. 2015. Compressiblity and penetrability of latossolo Vermelho-amarelo distrófico (oxisol) under varied Management systems and land uses. Revista Brasileira de Ciência do Solo, 39, 1, 86-93.

Pires, B. S.; Dias Junior, M. de S.; Rocha, W. W.; Araujo Junior, C. F.; Ribeiro, R. C. 2012. Modelos de capacidade de suporte de carga de um Latossolo Vermelho-amarelo sob diferentes usos e manejos. Revista Brasileira de Ciência do Solo, 36, 2, 635-642.

Raij, B. V.; Quaggio, J. A. 1983. Métodos de análise de solo para fins de fertilidade. Campinas: Instituto Agronômico de Campinas.

Reinert, D. J.; Reichert, J. M. 2006. Coluna de areia para medir a retenção de água no solo protótipos e teste. Ciência Rural, 36, 6, 19311935.

Richards. L. A. 1949. Methods of measuring soil moisture tension. Soil Science, 68, 1, 95-112.
Rocha, W. W.; Dias Junior, M. S.; Lima, J. M.; Miranda, E. V. V.; Silva, A. R. 2002. Resistência ao cisalhamento e grau de intemperismo de cinco solos na região de Lavras (MG). Revista Brasileira de Ciência do Solo, 26, 2, 297-303.

Roque, A. A. O; Souza, Z. M. de; Barbosa, R. S.; Souza, G.S. 2010. Controle de tráfego agrícola e atributos físicos do solo em área cultivada com cana-de-açúcar. Pesquisa Agropecuária Brasileira, 45, 7, 744-750.

Shwetha, P.; Varija, K. Soil water retention curve from saturated hydraulic conductivity for sandy loam and loamy sand textured soils. 2015. Aquatic Procedia, 4, 1, 1142-1149.

Snedecor, G. W.; Cochran, W. G. 1989. Statistical methods. 8th ed. Ames: Iowa State University Press.

Souza, I. A.; Ribeiro, K. G; Rocha, W. W.; Pereira, O. G.; Cecon, P. R. 2013. Physical properties of a Red-Yellow Latosol and productivity of a signalgrass pasture fertilized with increasing nitrogen doses. Revista Brasileira de Ciência do Solo, 37, 6, 1549-1556.

Stoessel, F.; Sonderegger, T.; Bayer, P.; Hellweg, S. 2018. Assessing the environmental impacts of soil compaction in Life Cycle Assessment. Science of The Total Environment, 630, 1, 913-921.

Veihmeyer, F. J.; Hendrickson, H. 1949. Methods of measuring field capacity and permanent wilting percentage of soils. Soil Science, 68, $1,75-94$.

Yang, F.; Zhang, G. L.; Yang, J. L; Li, D. C.; Zhao, Y. G; Liu, F.; Yang, R. M.; Yang, F. 2014. Organic matter controls of soil water retention in an alpine grassland and its significance for hydrological processes. Journal of Hydrology, 519, 3086-3093. 